INTRODUCING UNCERTAINTY OF AQUIFER PARAMETERS INTO AN OPTIMIZATION MODEI

Robert I. Ward and

Richard C. Peralta

1994 


\title{
INTRODUCING UNCERTAINTY OF AQUIFER PARAMETERS INTO AN OPTIMIZATION MODEL
}

by Robert I. Ward and Richard C. Peralta

\author{
Department of Biological and Irrigation Engineering \\ and \\ International Irrigation Center \\ Utah state University \\ Logan, UT 84322-4105 \\ (801) 797-2786 \\ FAX (801) 797-1248
}

REPORT IIC-94-1 
Table of Contents

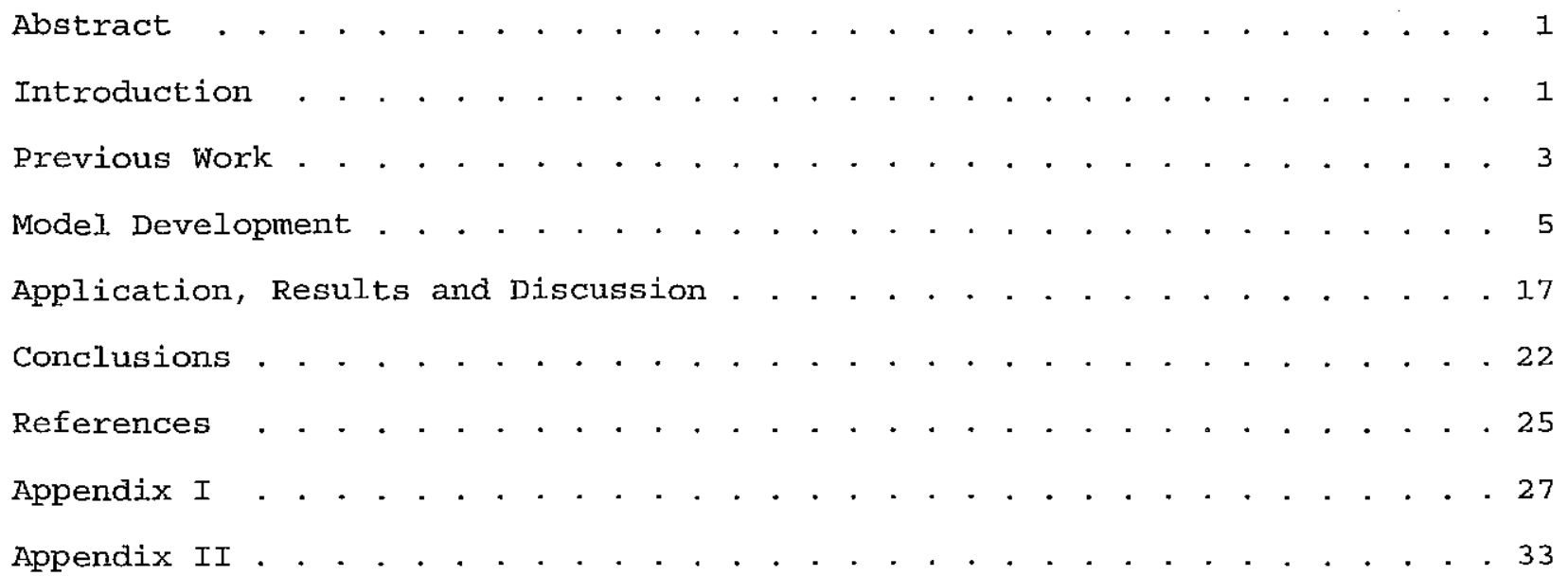




\section{List of Tables}

Table 1. Effect of Aquifer Parameter Uncertainty on 95\% Reliable Optimal Unsteady Pumping Strategy for Hypothetical Problem (run 1d) . 35

Table 2. Effect of Aquifer Parameter Uncertainty on $80 \%$ Reliable Optimal Unsteady Pumping Strategy for Hypothetical Problem (run 1d) . 36

Table 3. Summary of Trends Produced by Stochastic Analysis . . . . . . . 37 
List of Figures

Figure 1. Cross-section Demonstrating Sample Stochastic Constraints on Final Water Levels . . . . . . . . . . . . . . . . . . . . 38

Figure 2. Daily Pumping strategies for Increasing Uncertainty of Transmissivity as Compared to the Deterministic strategy (95\% Reliability Level) . . . . . . . . . . . . . . . . . 39

Figure 3. Daily Pumping strategies for Increasing Uncertainty of Effective Porosity as Compared to the Deterministic strategy (95\% Reliability Level) . . . . . . . . . . . . . . . . . . . . . . 40

Figure 4. Comparisons of Final Gradient and Total Pumping for the stochastic Runs at the $95 \%$ Reliability Level . . . . . . . . . . . . . . . 41

Figure 5. Comparisons of Final Gradient and Total Pumping for the stochastic Runs at the $80 \%$ Reliability Level. . . . . . . . . . . . . . . . 42 
A stochastic analysis is made for a previously described groundwater contaminant management model (Peralta and Ward, 1988). The stochastic model is based on incorporating uncertainty of the aquifer parameters cransmissivity and effective porosity into the model. This is accomplished by finding the partial derivative of drawdown with respect to each of these parameters using a Taylor series expansion approximation of the Theis equation. Input that is required for the stochastic version is the mean of the transmissivity and effective porosity, the coefficient of variation of the transmissivity and effective porosity, and a reliability level $(0 \%-100 \%)$. The reliability is a measure of the user's required confidence in the model solution. The user wants to be confident, at some probability, that the actual changes in head at pumping wells do not exceed the values calculated by the model, while, at the same time, he wants to be confident that actual changes in head at observation wells are at least as great as the calculated values. Thus, equations that are affected by heads at the observation wells are treated differently than equations that are affected by heads at the pumping wells.

Optimal strategies are presented to demonstrate sensitivity to changes in standard deviation of aquifer parameters and to changes in reliability level. Tests show that uncertainty of transmissivity affects the optimal pumping more and the final gradient and objective function less than uncertainty of effective porosity. In general, as uncertainty of aquifer parameters increases, optimal pumping values decrease, resulting in a poorer final hydraulic gradient. As the reliability level is increased optimal pumping decreases, again resulting in a poorer final gradient.

\section{Introduction}

Typically, some of the parameters of conceptual hydrologic models are calibrated using limited hydrologic information. The purpose of this paper is to describe how uncertain knowledge of aquifer parameters can be incorporated 
into an optimization program. The optimization program used as the basis for this paper has been described in detail (Peralta and Ward, 1988).

* The mathematical description of groundwater flow requires specific assumptions in order to fit the physical events into a set of equations for which a solution exists. The expression of the physical events by mathematical equations, the estimation of the aquifer parameters, and the approximation of complex analytical expressions by their discrete analog are important sources of error. Because these errors introduce uncertainty into groundwater modeling, future projections cannot be made with absolute certainty. The validity of these mathematical equations and the errors introduced by numerical methods have been discussed elsewhere. This work is only concerned with errors introduced by inaccuracies in aquifer parameters. In the practical simulation of real dynamic systems we are immediately faced with uncertainty as to exact physical parametexs. The investigator must establish tolerances within which the parameters of the physical system may vary without appreciably affecting the model results.

In light of the inherent randomness of subsurface flow and the existence of uncertainties in aquifer parameters, the groundwater flow system should be treated as a stochastic process and aquifer parameters be considered random variables. To carry this argument further, groundwater management models should, if possible, be able to consider the random nature of the subsurface flow system and derive management decisions accordingly.

In this paper, the development of a multi-period stochastic groundwater contaminant management model is illustrated using the Theis equation. The model considers explicitly the random characteristics of transmissivity and effective porosity in a confined aquifer. The stochastic management model is formulated by transforming the objective function and constraint equations containing random aquifer properties into a chance constrained expression which specifies the reliability requirements of the system performance (i.e. the user's confidence in the system results). 
PREVIOUS WORK

There have been very few studies that have used stochastic concepts at the macroscopic scale in subsurface flow models. The work that has been done can be placed into one of the three possible categories of uncertainty that have been investigated in model solutions. The three possible categories of uncertainty are: (1) those caused by measurement errors in the input parameters, (2) those caused by spatial averaging of the input parameters, and (3) those associated with the inherent stochastic description of nonhomogeneous or heterogenous porous media.

The error propagation study of Sagar and Kisiel (1972) falls into the first category. They investigated the influence of errors in initial head, transmissivity and effective porosity on the drawdown patterns predicted by the Theis equation for pumpage from a homogeneous isotropic confined aquifer. They utilized uniform frequency distributions for the input parameters, noting this is the usual Bayesian 'know nothing' prior distribution. They produced plots that show the growth through time of the percent error in bydraulic head at various radial distances form a pumping well with various input errors. They also concluded that a far more general and better method (yet mathematically complicated) of investigating error would be to consider the parameters as stochastic processes.

McElwee and Yukler (1978) looked at the sensitivity of groundwater models with respect to variations in transmissivity and effective porosity. They obtained sensitivity coefficients by finding the partial derivatives of the Theis equation with respect to each of these parameters. In general, they discovered that a $20 \%$ deviation in transmissivity or effective porosity can be handled adequately (error of less than 5\% of drawdown) by the first order formulation which is used in our paper.

The work of Bibby and Sunada (1971) combines aspects of approaches 1

and 2. In theix analysis they used a numerical simulation model of transient flow to a well in a confined aquifer. They utilized Monte Carlo simulation to 
investigate the effect on the solutions of normally distributed measurement errors in initial head, boundary heads, pumping rate, aquifer thickness, hydraulic conductivity and storage coefficient. In addition they analyzed the uncertainties introduced into the solutions by choosing spatially averaged parameter values at each grid point in the nodal mesh used in the numerical method. They assumed that within each nodal block, each input parameter (hydraulic conductivity, for example) can be represented by a general linear function that fully describes the spatial trends within the block. The uncertainties in the values of the coefficients of this general linear function (which are related to the number of available measurements) lead to uncertainty in the spatially averaged value used at each node in the simulation. This type of analysis leads to the normal distribution for the hydraulic conductivity values. This normal distribution identifies the approach as having more in common with the analysis of measurement errors (category 1) than with stochastically defined media (category 3) where hydraulic conductivity is usually recognized as being log-normally distributed.

A paper by Freeze (1975) falls into category 3 . He concluded that the most realistic representation of a nonuniform homogeneous porous media is a stochastic set of macroscopic elements in which the two basic hydrogeologic parameters (hydraulic conductivity and porosity) within these elements are assumed to come from frequency distributions.

Tung (1986) has developed a multi-period stochastic groundwater management model utilizing the Cooper-Jacob equation and the concept of unit response functions. His general conclusions were that effort should be given the better evaluate transmissivity and its variability. The effective porosity in a modeling process can be treated as deterministic and its accuracy is not critical. However, when the uncertainty of transmissivity is large the normality assumption for random drawdown may not be appropriate. Furthermore, the assessment of statistical properties of drawdown using first order analysis may not be appropriate. There have been some investigations regarding the appropriateness of first order analysis applied to situations where variation of 
system components is large.

Loaiciga and Marino (1987) develop a methodology for estimating the elements of parameter matrices in the governing equation of flow in a confined aquifer. The estimation techniques for the distributed parameters inverse problem include linear least squares and generalized least squares methods. Secondly, a nonlinear maximum likelihood estimation approach to the inverse problem is presented. The statistical properties of maximum likelihood estimators are derived, and a procedure to construct confidence intervals and do hypothesis testing is presented.

Model. Development

The Theis well function is the basic groundwater flow equation used by the simulation component of the management model. The deterministic version of the groundwater contaminant plume management model is used as the starting point for development of the stochastic management model. Some of the equations referred to are from the previous paper describing the deterministic model (Peralta and Ward, 1988). The new equations introduced in this paper are numbered as a continuation of this previous paper (i.e. there is only one equation no. I).

The goal. is to determine the optimal pumping rates for a specified planning horizon such that undesirable consequences do not occur. The stochastic approach allows the incorporation of uncertainty of aquifer parameters within the model. The model can utilize a probability distribution for each aquifer parameter. The model then will generate optimal pumping values that will produce no undesirable results for a specified reliability (confidence limit).

(1) Stochastic unit response function

The deterministic unit response function, $\delta$, can be obtained from a distributed parameter groundwater simulation model (Peralta and ward, 1988). However, when hydrogeologic information of an aquifer system is lacking or unavailable, a closed form analytical solution to an idealized condition can be 
utilized to derive a stochastic unit response function.

Since the unit response function characterizes an aquifer pumping-drawdown relationship, a groundwater management model can be very easily formulated once the response functions are defined. The deterministic management model does not consider the random nature of aquifer parameters. The stochastic model presented below has the same objectives, but incorporates probability in all equations that use unit response functions. probability is considered via information concerning the probability density function (pdf) of transmissivity (T) and effective porosity $(\varnothing)$.

Values for transmissivity and effective porosity are normally derived from a pump well test. Such a test provides in situ values of aquifer parameters averaged over a large and representative aquifer volume. Therefore, $\mathrm{T}$ and $\varnothing$ should be treated as random variables. Because the response function $\hat{\delta}$ is computed using the random variables $T$ and $\varnothing$, it too is random in nature.

The deterministic objective function equation (eq. 1), drawdown constraint equation (eq. 3) and the observation well potentiometric head constraint (eq. 4) are all functions of the probabilistic response function. Therefore, it is more appropriate and realistic to examine both objective function and constraints probabilistically; particularly when aquifer information is scarce.

In a stochastic environment, one wishes to specify limitations on allowable risk or required reliability of constraint performance. The necessary reliability for attaining the objective and satisfying the constraints can be represented by a confidence limit. 'This reliability states the models' confidence in the resulting potentiometric surface. The reliability can be determined based on the confidence of the model user in his estimates of aquifer parameters.

The following development is based on the procedure proposed by Tung (1.986) for the drawdown constraint. The restriction that actual drawdown (positive or negative change in head) in the field at any point $j$ at the end of the period $t$ resulting from pumping over the entire well field cannot exceed (or has to exceed) a specified value is the basis for the analysis. In this case the 
specified value is that calculated by the model. The drawdown is based on a specified reliability, $\rho$. In other words, we want to be $\rho \%$ sure that the computed value of the model will (or will not) be exceeded in the field. The computed drawdowns are themselves bounded so that within the model they will not exceed predetermined values.

For the drawdown constraint at pumping wells, there is a $\rho$ confidence that the actual drawdown at a pumping well will not exceed the $s_{j, t}$ drawdown value calculated by the stochastic model. Representing the actual drawdown using the relationship between drawdown and pumping (eq. 5) yields eq. 13a below. Rigorous testing of the validity of this constraint would be accomplished by:

(1) using a random number generator to create a large set of possible combinations of transmissivities and porosities,

(2) creating one set of $\delta$ for each combination developed in the previous step, and finally,

(3) using a eq.5 to compute the drawdowns that would result from using the optimal pumping strategy developed by the stochastic model.

If the sampling is large enough, $p \%$ of the drawdowns computed in this step should be less than the $\mathbf{s}_{\mathbf{j}, \mathrm{t}}$ computed by the stochastic model.

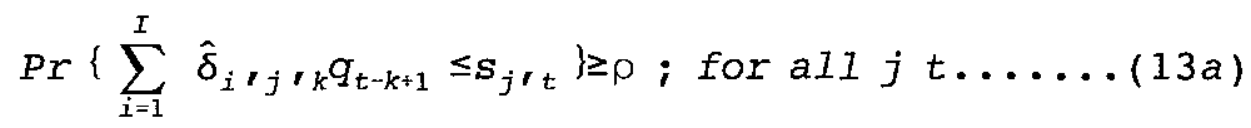

The calculated value, $s_{j, t}$ is limited by the drawdown constraint, eq. 3 . All such calculated drawdowns at pumping wells will be less than that specified by the drawdown constraint except for the drawdowns at the tightly constrained pumping wells. At such wells the stochastic drawdown will equal the constraint value. At the tightly constrained wells there is a $\rho$ probability that an actual drawdown is less than the stochastically created drawdown. At all other pumping wells the probability will be greater than $\rho$.

Heads at observation wells affect the objective function and constraint equation 4. There must be $\rho$ confidence that the actual drawdown at an 
observation well will be greater than the value, $s_{j, t}$, calculated by the stochastic model. However, to express this in the same form as eq. 13a, it is stated that there is a $1-\rho$ confidence that the actual drawdown will be less than that calculated by the stochastic model. This is expressed as:

$$
\operatorname{Pr}\left\{\sum_{i=1}^{I} \sum_{k=1}^{t} \hat{\delta}_{i, j, k} q_{t-k+1} \leq s_{j}, t\right\} \leq 1-\rho ; \text { for } a I 1 j \quad t \cdot \cdot(13 b)
$$

In equations $13 \mathrm{a}$ and $13 \mathrm{~b}$ the sequence of summation and notation for the increments $t$ and $t-k+I$ have been reversed from that in eq. 5. This provides a more clear derivation of the stochastic coefficients. This reversal has no effect on the final results.

A probabilistic statement of the drawdown constraint (or any statement where drawdown is used, such as the objective function) like eq. 13, is not mathematically operational, so further modification is needed. To make eq. 13 operational, it is necessary to assess statistical properties in random terms in this chance-constrained expression.

There have been a number of field investigations and laboratory experiments assessing the probability distribution of aquifer transmissivity and hyaraulic conductivity. Most findings indicate that the hydraulic conductivity has a log normal distribution. Because the response function, $\delta$, computed by the Theis equation, is a nonlinear function of transmissivity and effective porosity, the probability function of $\hat{\delta}$ as well as drawdown at any observation point cannot easily be determined. Therefore, a first-order analysis is used to estimate the statistical properties of the unit response function and drawdown at each observation point.

First-order analysis is a useful method to estimate statistical characteristics such as the mean and variance of a function involving random variables. In first order analysis, the function containing random variables is 
expanded in Taylor series about the mean values of random variables, i.e.

$$
\begin{gathered}
f(x)=f(u)+f^{\prime}(u)[x-x(u)]+\frac{f^{\prime \prime}(u)}{2 !}[x-x(u)]^{2} . . \\
. .+\frac{f^{n}(u)}{n !}[x-x(u)]^{n} \text {. . . . (14) }
\end{gathered}
$$

in which $f(x)$ is a function involving a random variable $x, f(u)$ is the mean value of $f(x)$ and $x(u)$ is the value of the random variable at the mean, $f(u)$.

Derivations of statistical of drawdown at each observation point, assuming independency of transmissivity and effective porosity, are given in Appendix I. Results are as follow:

$$
E\left(s_{j}, t\right)=\sum_{i=1}^{I} \sum_{k=1}^{t} \bar{B}_{i, j, k} q_{t-k+1} \ldots \ldots
$$

where $\bar{B}$ is the same as $\hat{\delta}$ in the deterministic model;

$$
\begin{aligned}
& \operatorname{var}\left(s_{j}, t\right)=\left[\sum_{i=1}^{I} \sum_{k=1}^{t} \overline{A_{i}}{ }_{j},{ }_{k} q_{t-k+1} s d t\right]^{2} \\
& +\left[\sum_{i=1}^{I} \sum_{k=1}^{t} \overline{P_{i}{ }_{j}, k} q_{t-k+1} s d s\right]^{2} \ldots \ldots
\end{aligned}
$$

in which $E\left(s_{j, t}\right)$ and $\operatorname{var}\left(s_{j, t}\right)$ are the mean and variance respectively of drawdown at observation point $j$ at the end of the $t$ period; sdt and sds are the standard deviations of the transmissivity and effective porosity respectfully and $B, A$

and $P$ are coefficients that are functions of the mean transmissivity and mean effective porosity. As can be seen in eq. 15, the mean drawdown is a linear function of pumping and represents the deterministic solution (50 percent 
reliability) but the variance (eq.16) is a quadratic function of pumping. Derivation of eqs. 15 and 16 enables the development of a deterministic equivalent for eqs. $13 \mathrm{a}$ and $13 \mathrm{~b}$. As shown in the next section, the equivalent is mathematically operational and permits explicit incorporation of random characteristics of the aquifer properties in the management model.

The total drawdown at any control point is the sum of the drawdown created by many individual pumps. Since drawdown is a random variable the central limit theorem applies. That theorem states that, if $n$ is large, a set of random variables has approximately a standard normal distribution. Therefore, the total drawdown at each observation point can be assumed to have a normal distribution with a mean and variance given by equations 15 and 16, respectively. Under the normality assumption the oxiginal chance constrained eqs. $13 \mathrm{a}$ and $13 \mathrm{~b}$ can be expressed as:

$$
\operatorname{Pr}\left\{Z \leq \frac{s_{j \prime n}-E\left(s_{j, n}\right)}{\sqrt{\operatorname{Var}\left(s_{j} \prime_{n}\right)}}\right\} \geq \rho \ldots . . . \cdot(17 a)
$$

for the drawdown constraint eq. 3 and

$$
\operatorname{Pr}\left\{Z \leq \frac{s_{j \prime_{n}}-E\left(s_{j \prime_{n}}\right)}{\sqrt{\operatorname{var}\left(s_{j n}\right)}}\right\} \leq 1-\rho \ldots . \cdots(17 b)
$$

for the objective function and constraint eq. 4 . $\mathrm{z}$ is a standard normal random variate with mean zero and unit variance. By substituting eq. 15 in to $17 \mathrm{a}$ and 17b, and since $F^{-1}[\rho]=-F^{-1}[1-\rho]$, an equivalent expression can be written as:

$$
\sum_{i=1}^{I} \sum_{k=1}^{t} \bar{B}_{i \prime j \prime k} q_{t-k+1} \pm \sqrt{\operatorname{var}\left(s_{j \prime t}\right)} F^{-1}[\rho] \leq s_{j \prime t}
$$

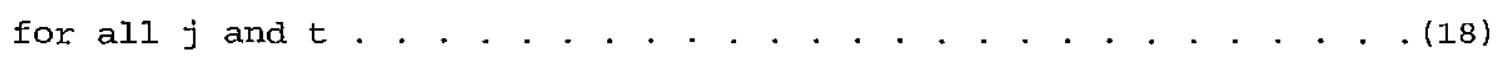
in which $F^{-1}[\rho]=$ a standard normal deviate corresponding to the normal 
cumulative distribution function of $\rho$. The plus sign on the left side of the equation produces the equation stating that there is a $\rho$ probability that the actual drawdowns at pumping wells are less than the calculated value, $s_{j, t}$. The minus sign produces the equation stating that there is a $1-\rho$ pobability that the actual drawdowns at observation wells are less than the calculated value.

Note that the second term in eq. 18 involves a square root of the variance of drawdown at each observation point which, in turn, is a quadratic function of unknown decision variables $q$. The deterministic equivalent of a chanceconstrained equation in nonlinear. Standard linear programming codes cannot solve problems with nonlinear constraint equation. However, as suggested by Tung (1986), quasi-linearization can be employed to linearize the nonlinear term in eq. 18 .

This linearization is actually a txial and errox method using an "estimate" of the optimal pumping to determine the stochastic coefficients. The iterative process is shown as a flow chart called Figure $I$ in Tung (1986). In the process of linearization, the nonlinear term in eq. 18 is expanded as a Taylor series (i.e. eq. 13) about this estimate of optimal pumping, $Q \mathrm{O}_{\mathrm{t}-\mathrm{k}+1}$.

$$
\begin{aligned}
& f(q)=\sqrt{\operatorname{var}\left(s_{j \prime t}\right)} \approx f(Q O)+\sum_{i=1}^{I} \sum_{k=1}^{t}\left\{\left[\frac{\partial f(q)}{\partial q_{t-k+1}}\right]_{Q o_{t-k \neq 1}}\right. \\
& \left.\left.{ }_{t-k+1}-Q O_{t-k+1}\right]\right\}+H O T \text {. . . . . . . . . . . . . . . . . (1 }
\end{aligned}
$$

in which HOT are the higher order terms. After neglecting the higher order terms and some algebraic manipulations, the first-order linear approximation of the nonlinear terms (derived in Appendix II) can be expressed as:

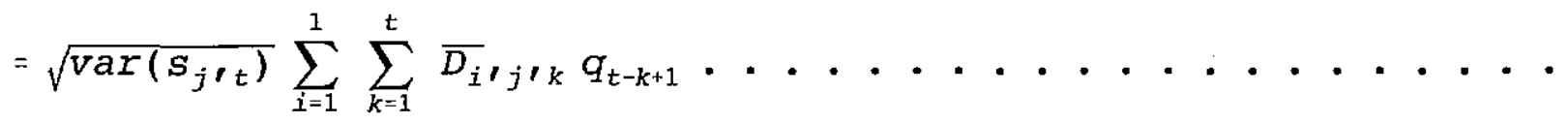

whexe: 


$$
\begin{gathered}
\frac{1}{f(Q O)}\left[f t(Q O) \overline{A_{i} \prime^{\prime}{ }^{\prime}} s d t+f s(Q O) \overline{P_{i} j^{\prime} k} s d s\right] \ldots \ldots \\
f(Q O)=\sqrt{f t(Q O)^{2}+f s(Q O)^{2}}
\end{gathered}
$$

$$
\begin{aligned}
& f t(Q O)=\sum_{i=1}^{I} \sum_{k=1}^{t}\left[\overline{A_{i}}, j, k Q O_{t-k+1}\right] s d t \\
& f s(Q O)=\sum_{i=1}^{I} \sum_{k=1}^{t}\left[\overline{P_{i}}, j, k Q O_{t-k+1}\right] s d s
\end{aligned}
$$

sdt is the standard deviation of transmissivity

sds is the standard deviation of effective porosity

$\widetilde{\mathrm{A}}$ and $\overline{\mathrm{P}}$ are defined by equations 28 and 30 respectively.

Finally, substituting eq. 20 into eqs. $18 \mathrm{a}$ and $18 \mathrm{~b}$ results in a linear approximation for the stochastic equivalent to the original deterministic constraint on drawdown:

$$
\sum_{i=1}^{I} \sum_{k=1}^{1} E_{i, j, k} q_{t-k+1} \leq s_{j, t} \cdot \ldots . \cdots \cdot \ldots . \cdots \cdot \ldots
$$

where:

$$
E_{i \prime j \prime k}=\overline{B_{i} \prime^{\prime} k}+F^{-1}[\rho] \overline{D_{i}{ }^{\prime} \prime^{\prime} k}
$$

for drawdown constraint equation 3 and 


$$
E_{i \prime j \prime k}=\overline{B_{i} \prime^{\prime}{ }^{\prime} k}-F^{-1}[\rho] \overline{D_{i} \prime j \prime k}
$$

for the objective function and constraint equation 4.

Checking the signs for the $\bar{B}$ and $\bar{D}$ coefficients reveals that the stochastic unit influence coefficient (E) responds the same whether showing the influence of an injection wells both $\bar{B}$ and $\bar{D}$ are negative values. Therefore $\mathrm{E}$ is larger in absolute magnitude than the deterministic unit influence coefficient for the drawdown constraint. E is smaller than the deterministic coefficient for the objective function and constraint eq. 4. At extraction wells both ${ }^{-} B$ and $D$ are positive; producing a larger absolute value for $\mathrm{E}$ in the drawdown constraint and a smaller value for the objective function and constraint eq. 4 .

To convert the original deterministic model into a stochastic model replace the drawdown constraint eq. 3 with eq. 22 and use $E_{i, j, k}$ for $\hat{\delta}_{j} i, j, k$ in the objective function. Clearly, $E_{i, j, k}$ can be considered as a stochastic unit response function derived from the Theis equation. And it should be noted that the deterministic model actually represents a reliability of .50 (when $\mathrm{F}^{-1}[.50]=0$ ).

(2) Reliability determination

There are drawdown terms (for observation wells) in the objective function and constraint eq. 4 as well as in drawdown constraint eq. 3 (for pumping wells). Reliability is treated differently in the two cases. Refer to Figure 1 during the following discussion.

Let's assume a reliability level of 0.95. In a drawdown constraint one wishes to be 95 percent sure that the change in water level does not exceed the prespecified maximum change (i.e. does not violate predetermined bounds on head). One used the standaro normal deviate $\left(F^{-1}[\rho]\right)$ corresponding to a reliability of 0.95 for the drawdown constraint (i.e. $F^{-1}[.95]=1.64$ ). The procedure described 
previously computes a stochastic unit response coefficient for the 95 percent confidence level. The coefficient is larger than a deterministic coefficient (which corresponds to a 50 percent confidence level). Since a unit pumping influence coefficient less pumping is feasible before drawdown constraints become tight.

When considering the objective of raising water levels to prevent contaminant movement one wishes to be 95 percent confident that head changes equal or exceed calculated values. Therefore, with the objective function and constraint 10 one uses the standard normal deviate corresponding to a reliability of .05. This produces stochastic influence coefficients that are numerically smaller than 95\% of all deterministic influence coefficients. For identical pumping values the $95 \%$ probability change in water levels needed to achieve a horizontal gradient is much greater than that needed using deterministic coefficients. This guarantees that pumping values calculated by the model are equal to or greater than those required by the deterministic model to produce a horizontal gradient.

However, this guarantee also allows constraint eq. 4 (which specifies that final heads at down-gradient observation wells are greater than final head at source) to force the objective function value to be larger than an objective function value resulting from only trying to minimize the head differences between the contaminant source and all observation wells. Greater pumping values may actually cause the heads at the down-gradient observation wells to 'overshoot' the head at the source and produce a reverse gradient. This is demonstrated in the Application Section where the objective function and reverse gradient increase as aquifer parameter uncertainty increases. The 'tight' downgradient observation well is the one whose final head is equal to the final head

at the source. AlI other down-gradient observation well heads are higher than the source head, therefore, producing a larger objective function value.

(3) Determination of aquifer parameters

Estimation of transmissivity and effective porosity has received much 
attention in the literature in recent years and was discussed in the Review of Literature section. From eqs. 15 and 16 it is seen that the mean and variance of transmissivity and effective porosity are needed in the stochastic version of the optimization model. Mary methods for determining these statistics are described in the literature. Here a Bayesian approach is used to derive the mean and variance for transmissivity and effective porosity.

The Bayesian approach uses a prior (also called unconditional) probability distribution function (pdf) and a likelihood pdf to determine the mean and variance for the aquifer parameters. This mean and variance describe the posterior or conditional pdf used within the stochastic model. The prior pdf is based on knowledge of the aquifex obtained from past experience. This study suggests using aquifer material (soil type) as the basis for the prior pdf. The likelihood pdf is developed from current information (field or lab data) about the aquifer in question.

In the stochastic analysis portion of this study the standard deviation of transmissivity and effectie porosity are varied to determine how these changes affect the objective function value. However, in a real situation, one would estimate a mean and variance for these aquifer parameters from a prior pdf and a 'likelihood' pdf. The user would select a description of the soil type from a given list. Based on a range of values of transmissivity and effective porosity associated with each soil type (derived from numerous references), a

prior pdf mean ( $\left.\vec{X}_{0}\right)$ and variance (Vo) are determined. This determination is made by assuming that the range of values spans three standard deviations each side of the mean (99\% confidence interval). With this assumption and assuming a log-normal pdf for transmissivity and a normal pdf for effective porosity one can compute the mean and standard deviation. If there are no field data values for the problem the prior pdf becomes the posterior pdf.

If one has field data values, the mean ( $\mathrm{X}$ ) and variance (V) are determined using standard equations for mean and variance of a data population. This mean and variance for the field data values define the likelihood $p$ [df. The mean and 
variance for transmissivity are calculated using the natural log of all transmissivity values because these values are known to be normally distributed. The posterior pdf is related to the prior pdf and likelihood pdf as shown:

posterior distribution $\infty$ prior distribution $x$ likelihood distribution

The mathematics of multiplying a normally distributed likelihood pdf by a normally distributed prior pdf has been previously derived (Iindley, 1970 ) Assuming the natural log data values for transmissivity and the data values for effective porosity are normally distributed, the posterior mean, $E()$, and posterior variance, var( ) for either parameter are calculated from:

$$
\begin{aligned}
& E()=\frac{1}{V O^{-2}+V^{-2}}\left(V O^{-2} \bar{X} O+V^{-2} \bar{X}\right] \cdot \cdot \cdot \cdot \cdot \cdot(23 a)
\end{aligned}
$$

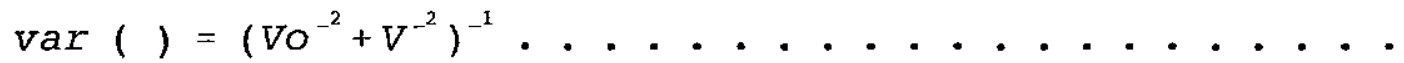

The expected value, $\mathrm{E}$, and the variance, var, for effective porosity are used as the posterior mean and variance. However, because natural log values are used to determine the expected value and variance for transmissivity, these values must be converted back to represent the mean and variance of the actual transmissivity values. Standard equations for the mean and variance of a population which has a log normal pdf and the expected value and variance of its natural log values are known are used (Johnson and Kotz, 1970). These are:

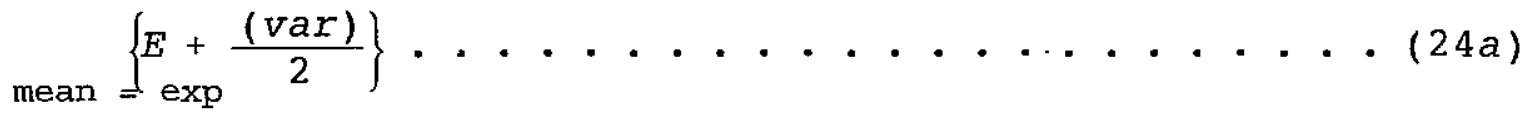
$\operatorname{var}\{\{\operatorname{axp}[=(\operatorname{var})+2 E]\}\{\exp [(\operatorname{var})]-1\} \cdot \cdot \cdot \cdot \cdot \cdot \cdot . \cdot \cdot \cdot \cdot(24 b)$ 
These two equations are used assuming the entire population of values is available. Since the prior pdf uses the knowledge of a large amount of data for each soil type this assumption is sound.

Application, Results and Discussion

The stochastic optimization model was applied to the same hypothetical groundwater contamination problem that was used to analyze the deterministic version. Aquifer parameters' (transmissivity and effectie porosity) coefficient

of variation (ratio of standard deviation to mean) and required solution reliability were varied in consecutive runs.

The simulation component and optimization component were run on an IBM AT with $640 \mathrm{~K}$ bytes of RAM, a 30 MEG internal hard disk with a floppy disk drive, and math coprocessor.

Utilized physical parameters for model run ld include a transmissivity of $1255 \mathrm{~m}^{2} / \mathrm{d}\left(13,500 \mathrm{ft}^{2} / \mathrm{d}\right)$, and an effective porosity of 0.3 . The original hydraulic gradient was $0.54 \%$. Maximum and minimum acceptable pumping rates, based on available equipment, are $135 \mathrm{~L} / \mathrm{s}$ and $0 \mathrm{~L} / \mathrm{s}$. This was based on the performance curve for a pump that can discharge $150 \mathrm{~L} / \mathrm{s}$ against $6 \mathrm{~m}$ of head at $80 \%$ efficiency. The upper limit on head at all injection wells was the ground surface (5.8 $\mathrm{m}$ above the initial water table). This should prevent pressurized injection (Lefkoff and Gorelick, 1986). The lower limit on head at extraction wells prevented such changes in tansmissivity that would invalidate the use of superposition. In general, if the change in transmissivity is less than 10 percent, the aquifer can be treated as a confined aquifer system.

The stochastic model was applied to the same hypothetical system described for the deterministic model in the previous paper (Peralta and Ward, 1986). Results are shown in Tables 1 and 2 for comparison with the deterministic model (run 1d). The coefficients used for this analysis were $W_{f}=1.0$ and $c^{5}$ and $c^{H}$ equal 
to their original values. Therefore, the results shown for this analysis are for a strongly hydraulic objective function.

The initial pumping (QO) used in the iterative solution procedure of the stochastic model was the optimal pumping from the deterministic model run. It was found that two iterations brought acceptable agreement (convergence within 5 percent) between the "estimated" pumping values and the final optimal pumping. The weight factor in the objective function was adjusted for identical runs as was described in the previous paper (Peralta and Ward, 1988), but as was found then, all weight factors of 1.0 and greater produced the same results. Subsequent tests used a weight factor of 1.0 .

In all, ten stochastic optimizations were pexformed. These utilized a range of values for the coefficient of variation (CV) for both transmissivity and effective porosity and used two reliabilities (a constant for all wells and all time periods for each run). Figures 2 and 3 graphically depict the pumping strategies developed for the five stochastic model runs made at the 95 percent reliability level. Figure 2 shows the pumping trends as the uncertainty of transmissivity increases from run 1 s to run $3 \mathrm{~s}$ as compared to the deterministic run (Id). Figure 3 shows the pumping trends as uncertainty of effective porosity increases from run $1 \mathrm{~s}$ to runs $4 \mathrm{~s}$ and $5 \mathrm{~s}$. The same general pumping trends are evident for the runs made at the $80 \%$ reliability level.

To analyze the predictability of these results we look first at the equation for the stochastic influence coefficient $\mathrm{E}$ (eq. 22) and reference Figure 1. From a table of standard normal deviates it is known that, as reliability $\left(\rho=F(z)\right.$ ) increases, $\mathrm{z}$ (which equals $F^{-1}[\rho]$ ) increases. Therefore, looking at equation 22 we see that, as reliability increases, E for the objective function and constraint equation 4 decreases and $\mathrm{E}$ for the drawdown constraint increases. In addition, as uncertainty of aquifer parameters increases (increasing CV), the standard deviation of the parameters increases; thereby increasing the value of $\bar{D}$ (eq. 21). In summary, an increase in uncertainty of aquifer parameters produces the same result as an increase in reliability; smaller $E$ for the objective function and constraint equation 4 and larger $\mathrm{E}$ for the drawdown 
constraint.

As stated, for the drawdown constraints, increasing reliability or uncertainty of parameters produces a larger influence coefficient. This causes a greater reaction of the potentiometric surface to a unit of pumping. Therefore, this increase allows for less pumping during a unit of time because the upper bound on drawdown is reached more quickly. In the case of a reliability of .95 we know the $F^{-1}$ [.95] value (1.64) is equal to or larger than 95 percent of all $\mathrm{F}^{-1}[\rho]$ values; thus the $\mathrm{E}$ value for a reliability of .95 is equal to or greater than 95 percent of $\mathrm{E}$ values for the same aquifer parameters. This confirms the stochastic constraint that in the field the upper bound on drawdown will not be exceeded 95 percent of the time. Tables 1 and 2 reflect the trend of increasing reliability or increasing uncertainty of parameters and the resulting decrease in allowable pumping.

Why, then, does the pumping increase for the last time period or are there more time periods of pumping as reliability or CV increases? While the large coefficients are causing large head increases at the injection wells (thus restricting the amount of pumping) the small stochastic influence coefficients for the objective function and constraint equation 4 cause much smaller reaction of the potentiometric surface at the observation wells. Thus, lower pumping values caused by increasing the reliability or uncertainty have even a smaller effect on drawdown at the observation wells. Yet the goal is still to minimize the objective function. To do this, additional pumping periods are needed or more pumping is required during the last time period as reliability or uncertainty increases. This trend is shown in Tables 1 and 2. The objective function uses the large drawdowns at the pumping wells to calculate pumping costs; thus producing the highest costs. The objective function uses the small drawdowns at the observation wells to determine the differences in head; thus producing a large sum of head differences. Thus we are assured that the objective function value is the largest expected for the given input and that the results in the field will probably not exceed the calculated value.

However, constraint equation 4, because it uses the smaller E values for 
the observation well head calculations, actually causes the hydraulic gradient to "overshoot" horizontal. The smaller E values produced at the .05 reliability level for observation well head calculations give us a 95 percent confidence that the heads are those calculated (using these $\mathrm{E}$ values) or greater; thus causing the reverse gradient. Remembering that the final gradients are always reverse gradients, Tables 1 and 2 show that as reliability or uncertainty increase the final gradient is larger in the reverse direction. The confidence in the final gradient is further complicated by the fact that the target elevation (normally the head at the contaminant source) is itself stochastic. Therefore, the actual reliability of the final gradient will be something less than the specified value; but that reliability cannot be determined with precision.

Table 3 summarizes the trends that developed as uncertainty of aquifer parameters and reliability were systematically varied. Figures 4 and 5 graphically show the trends in total pumping and the resulting final gradient. Figure 4 shows the five stochastic runs using a reliability of 95 percent normalized to the deternistic run (1d). Figure 5 shows the five runs using a reliability of 80 percent. As the coefficient of variation (CV) for transmissivity increases (runs $1 \mathrm{~s}, 2 \mathrm{~s}$ and $3 \mathrm{~s}$ ) the influence coefficients for the drawdown constraint increase and those for the objective function decrease. The expected result is decreased pumping for each time period (but larger total pumping), increased final average gradient and objective function value.

Runs Is, 4s and 5s show the results of increasing the CV for the effective porosity while holding the transmissivity CV constant. The general trend for these runs is the same as those for runs $1 \mathrm{~s}, 2 \mathrm{~s}$ and $3 \mathrm{~s}$. The resulting gradient and objective function for runs $4 \mathrm{~s}$ and $5 \mathrm{~s}$ show a sharp increase from run $1 \mathrm{~s}$. The increased CV produces larger influence coefficents for the drawdown constraint and smaller coefficients for the objective function just as the increased CV for transmissivity does. However, the changes in these coefficients are small compared to those produced by comparable incrases in transmissivity CV; and cause only small differences in pumping between xuns $1 \mathrm{~s}, 4 \mathrm{~s}$ and $5 \mathrm{~s}$. In comparison, the 
resulting gradient and objective function are much worse than those resulting from comparable transmissivity changes in runs $2 \mathrm{~s}$ and $3 \mathrm{~s}$.

To explain this dfference we look at the difference in sign between the $\bar{A}$ coefficients (eq. 28) which are affected by changes in transmissifity CV and the $\overline{\mathrm{P}}$ coefficients (eq. 30 ) which are affected by changes in effective porosity $\mathrm{CV}$. The negative sign with the $\overline{\mathrm{P}}$ coefficient indicates it will affect the optimal strategy in an opposite manner than that of the $\bar{A}$ coefficient. As the $\mathrm{CV}$ of transmissivity is increased, there is a large change in pumping and a small change in gradient and objective function. For the same CV increase in effective porosity there is a small change in pumping and a large change in gradient and objective function. The two parameters (transmissivity and effective porosity) cause an opposite relationship between pumping and its effect on the objective function and the constraints.

Table 2 displays results of the same variation in the $\mathrm{CV}$ of the two parameters, computed using a reliability level of 0.80 . As expected, the reduction in reliability increases the optimal pumping values and improves the final gradient and objective function. The smaller reliability produces smaller stochastic unit response coefficients. Resulting strategies and water levels are more similar to those from the deterministic model (reliability $=0.50$ ) than are those developed using a 0.95 reliability.

Strategies for runs $4 \mathrm{~s}$ at the 95 percent reliability level and $5 \mathrm{~s}$ at 80 percent reliability, have no pumping on day 7 and yet require pumping on day 8 . This is a definite change in the overall pattern of the stochastically optimal pumping strategies. However, a look at the sensitivity values for the pumping during days 7 and 8 gives an indication that it is not a major change. The sensitivity value (amount the objective function would change with a unit increase in pumping during that day) associated with each pumping value for days 7 and 8 for those two runs are very small. For example, these sensitivities are in the range of $10^{-4}$ to $10^{-15}$ as compared to a sensitivity of 0.7 to 1.3 for the tight pumping value in most other runs. This indicates that the pumping for day 8 could also be 0 without any significant change in the objective function. 
Therefore, the 0 pumping for day 7 and a pumping value for day 8 of these runs could be 0 pumping for both days 7 and 8 without a dramatic change in the overall pattern of the results.

Comparisons to Tung's (1986) analysis are difficult to make because his objective function was to maximize pumping which is not affected by the stochastic influence coefficient. The only constraint was on drawdown. In addition, the Cooper-Jacob equation (which is only appropriate for small values of the Boltzman variable; $u \leq 0.01$ ) used to derive the stochastic unit influence coefficient shows $\overline{\mathrm{p}}$ to be equal to 0 except for the first time period. However, the general trends Tung speaks of concerning transmissivity apply to this analysis: 1) Pumping increases as reliability or CV decreases and 2) Uncertainty of transmissivity causes a larger change in pumping than does a comparable change in effective porosity. However, this study indicates effective porosity has an effect on the drawdown at the observation wells (something Tung considers negligible) and hence has an effect on the objective function value. In addition, the daily pumping increases with decreasing effective porosity cV but, at the same time, the total pumping decreases. In summary, the trends shown in this analysis are found in Table 3 .

\section{Conclusions}

A procedure developed by Tung (1986) was used to incorporate uncertainty of aquifer parameters into our model. A stochastic unit response function (E, based on the Theis wel.1 function) was developed and used in the same manner as the unit response function in a deterministic model. This $\mathrm{E}$ value is dependent upon the uncertainty of aquifer parameters as measured by the coefficient of variation and a specified reliability of the solution.

Drawdown at observation wells (which affect the objective function and gradient constraints) must be treated differently than drawdown at the pumping wells (which affect the drawdown constraint). For example, if a reliability of 95 percent is specified for our solution, an $\mathrm{E}$ value corresponding to a reliability of .95 is used for the drawdown constraint because the user wants to 
be 95 percent confident that the resulting drawdown produced by the optimal pumping at the pumping wells is the value calculated or less. Whereas, the E value corresponding to a reliability of .05 is used to determine drawdown at the observation wells because the user wants to be 95 percent confident the actual drawdown (produced by the optimal pumping) at the observation wells equals or exceeds the calculated value. This means E values corresponding to a reliability of .95 are used for the drawdown constraint and those corresponding to a reliability of .05 are used with the objective function and constraint equation 10 .

This theory guarantees the user a 95 percent confidence level. for the drawdown constraint. However, because the objective function minimizes the head differences between the observation wells (whose values are stochastic) and the source (whose value is also stochastic) a joint 95 percent confidence level cannot be guaranteed. It would be some value slightly less than 95 percent and cannot readily be determined.

The major differences between Tung's analysis and this study are:

1) Tung used the Cooper-Jacob equation to derive the stochastic coefficients and 2) Tung's objective function was to maximize pumping and therefore, the objective function did not incorporate stochastic coefficients.

The study results shown in Tables 1 and 2 agree in general with the conclusions of Tung. As the reliabjlity level decreases or as aquifer parameter uncertainty decreases, the pumping for each time period increases. As a consequence the objective function improves.

The results of changes in uncertainty of effective porosity differ from those of Tung. Tung's derivation of the $\overrightarrow{\mathrm{P}}$ coefficient (the partial derivative of drawdown with respect to effective porosity; equation 30 ) using the Cooper-Jacob equation showed it to have a value of 0 for all time periods except the first time period. Therefore, changes in uncertainty of effective porosity had almost no effect on the optimal pumping values. This may be due to the fact * that the Cooper-Jacob equation is only valid for small values of the Boltzman variable $(u \leq .01)$. Our study shows the $\overline{\mathrm{p}}$ coefficient to have values for all 
time periods. For equal changes in COV, effective porosity produces smaller changes in pumping than does txansmissivity. However, the resulting final gradients produced by these small changes in pumping are much poorer than the final gradient produced by a comparable change in cov of sransmissivity. These results indicate that uncertainty in effective porosity has little effect on allowable pumping as Tung concluded, but the final gradient is affected in an adverse way.

Three general statements can be made from the stochastic analysis of this model :

1. Introducing stochasticity into the optimization model increases the value of the objective function.

2. Lowering the reliability level produces a model which allows more pumping (increased $O \& M$ cost) and produces an improved final gradient.

3. Changes in uncertainty of transmissivity and effective porosity both produce the same general changes in optimal daily pumping and final gradient.

4. Changes in uncertainty of transmissivity and effective porosity produce opposite effects on the total optimal pumping required. 


\section{References}

1. Bear, J. 1979. Eydraulics of Groundwater. McGraw-Hill, New York.

2. Bibby, R., and Sunada, D. K. 1971. Statistical error analysis of a numerical model of confined groundwater flow. Stochastic Hydraulics: Proceedings First International symposium on Stochastic Hydraulics, edited by C. I. Chiu, pp. 591-612.

3. Dagan, G. 1985. Stochastic modeling of groundwater flow by unconditional and conditional probabilities: the inverse problem. Water Resources Research, Vol. 21, No. 1, January, pp. 65-72.

4. Freeze, R. A. 1975. A stochastic-conceptual analysis of one dimensional groundwater flow in nonuniform homogenous media. Water Resources Research, Vol. 11, No. 5, October, pp. 725-740.

5. Freeze, R. A. and Cherry, J. A. 1979. Groundwater. Prentice-Hall, Inc., Englewood Cliffs, New Jersey.

6. Greenberg, M. D. 1978. Foundations of Applied Mathematics. Prentice-Hall Inc., New Jersey.

7. Lindley, D. V. 1970. Bayesian Statistics, A Review. Society for Industrial and Applied Mathematics.

8. Loaicigia, H. A. and Marino, M. A. 1987. The inverse problem for confined aquifer flow: indentification and estimation with extensions. Water Resources Research, Vol. 23, No. 1, January, pp. 92-104. 
9. McElwee, C. D., and Yukler, M. A. 1978. Sensitivity of groundwater models with respect to variations in transmissivity and storage. Water Resources Research, Vol. 14, No. 3, June, pp. 451-459.

10. McWhorter, D., and Sunada, D. K. 1977. Groundwater Hydrology and Hydraulics, Water Resources Publications, Colorado.

11. Murtagh, B. A. and Saunders, M. A. 1983. MINOS 5.0 Users Guide. Technical Report sOL 83-20, stanford Univ., California.

12. Peralta, R. C. and Ward, R. L. 1986. Optimal piezometric surface management for groundwater contaminant control. Copies may be obtained by writing ASAE. Paper no. 86-2513. Presented at ASAE Winter meeting, Chicago, 1986 .

13. Sagar, B., and Kisiel, C. C. 1972. Limits of deterministic predictability of saturated flow equations. Proceedings of the second Symposium on Fundamentals of Transport Phenomena in Porous Media, Vol. 1, International Association of Hydraulic Research, Guelph, Canada, pp. 194-205.

14. Todd, D. K. 1980. Groundwater Hydrology, second edition. John Wi.ley \& Sons, Inc., New York.

15. Tung, Y. K., 1986. Groundwater Management Chance-Constrained Model. Journal of Water Resources Planning \& Management, Vol. 112, No. 1, January, pp. 1-19. 
APPENDIX I - Analysis of uncertainty in drawdown

Discrete formulation of drawdom at observation point $j$ at the end of the nth period is given by eq. 11 as:

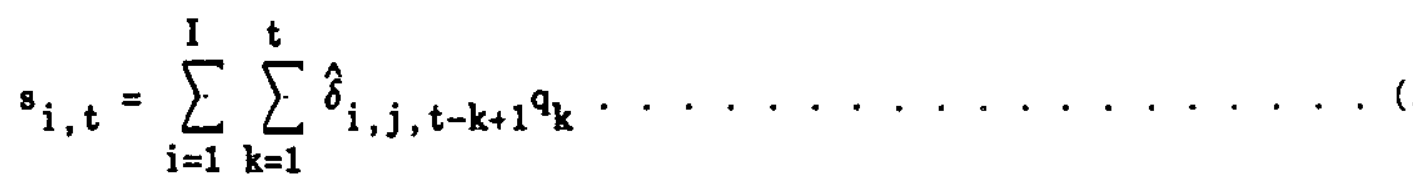

where $\hat{\delta}_{i, j, t-k+1}=$ the unit response function which can be derived from the Theis equation as:

$\hat{\delta}_{i, j, k}=\frac{1}{4 \pi T}\left\{\mathbb{W}\left[u_{i, j, k}\right]-W\left[u_{i, j, k-1}\right]\right\}$

where:

$$
u_{i, j, k}=\frac{r^{2} \phi}{4 T k}
$$

and

$$
\nabla\left[u_{i, j, k}\right]=\int_{u}^{\infty}\left[\frac{e^{-v}}{v}\right] d v
$$

Since $T$ (transmissivity) and $\phi$ (effective porosity) are random variables, the unit response function as well as drawdown are both random variables because they are functions of random variables.

To estimate statistical properties of random variables, the first-order analysis of uncertainty is ewployed. Taylor's expansion of dramdown about the mean values of $T$ and $\phi$ can be expressed as: 


$$
s_{i, t}=\sum_{i=1}^{1} \sum_{k=1}^{t} \bar{B}_{i, j, k^{q} t-k+1}+\left.\frac{\partial s_{i, t}}{\partial \bar{T}}\right|_{\bar{T}}(T-\bar{T})+\left.\frac{\partial s_{i, t}}{\partial \phi}\right|_{\bar{\phi}}(\phi-\bar{\phi})+H O T
$$

where $\bar{B}_{i, j, k}$ is computed using mean values $\bar{T}$ and $\bar{\phi}$ and HOT

represents the higher order terms. The time increments of $k$ and $t-k+1$ are reversed from those in eq. 5 but they produce the same result.

First, we compute the middle tera on the right hand side. The first order partial derivative of $s_{i, t}$ with respect to $T$ can be obtained by Leibnitz rule for differentiating an integral (Greenberg, 1978, page 18):

$$
I^{\prime}(c)=\int_{a(c)}^{b(c)} \frac{\partial f(x, c)}{\partial c} d x+f[b(c), c] \frac{d b}{d c}-f[a(c), c] \frac{d a}{d c} \ldots \ldots . . .(26)
$$

Performing the mathematics of the differentiation in three parts we define:

$$
I^{\prime}(c)=\frac{\partial s_{j, t}}{\partial T}=\frac{\partial}{\partial T}\left[\sum_{i=1}^{I} \sum_{k=1}^{t} \hat{\delta}_{i, j, k} q_{t-k+1}\right]
$$

For the first term on the right hand side of (26): 


$$
\begin{aligned}
\int_{a(c)}^{b(c)} \frac{\partial f(x, c)}{\partial c} d x & =\int_{u}^{\infty}\left\{\partial\left[\frac{1}{4 \pi T}\left(\frac{e^{-v}}{v}\right)\right] / \partial T\right\} d v \\
& =-\frac{1}{4 \pi T^{2}} \int_{u}^{\infty}\left[\frac{e^{-v}}{v}\right] d v
\end{aligned}
$$

Second term:

$$
f[b(c), c] \frac{d b}{d T}=0 \text { because } b=\text { constant }(\infty)
$$

Third term:

$$
f[a(c), c] \frac{d a}{d T}=\frac{1}{4 \pi T}\left[\frac{e^{-u}}{u}\right] \frac{d u}{d T} \text { where } \frac{d u}{d T}=\frac{d\left[\frac{r^{2} \phi}{4 T k}\right]}{d T}=-\frac{r^{2} \phi}{4 T^{2} k}=-\frac{u}{T}
$$

therefore:

$$
f[a(c), c] \frac{d a}{d T}=\frac{1}{4 \pi T}\left[\frac{e^{-u}}{u}\right] \frac{-u}{T}=-\frac{1}{4 \pi T^{2}} e^{-u}
$$

Adding the three terms:

$$
\begin{aligned}
& \frac{\partial s, j, t}{\partial T}=\sum_{i=1}^{I} \sum_{k=1}^{t} \bar{A}_{i, j, k^{q_{t-k+1}} \ldots \ldots} \ldots . . . . \\
& \text { in which: } \\
& \bar{A}_{i, j, k}=\frac{1}{4 \pi T^{2}}\left\{e^{-u_{k}}-\int_{u_{k}}^{\infty}\left[\frac{e^{-v}}{v}\right] \text { dv }\right\} \quad \text { at } k=1 ;
\end{aligned}
$$




$$
=\frac{1}{4 \pi T^{2}}\left\{e^{-u_{k}}-e^{-u_{k-1}}+\int_{u_{k}}^{u_{k}-1}\left[\frac{e^{-v}}{v}\right] d v\right\} \text { at } k>1 \ldots . . \text { (28) }
$$

Similarly, the first-order partial derivative of drawdown with respect to the effective porosity can be obtained in three parts from Leibnitz rule:

For the first term on the right hand side of (26):

$$
\int_{a(c)}^{b(c)} \frac{\partial f(x, c)}{\partial c} d x=\int_{u}^{\infty}\left\{\partial\left[\frac{1}{4 \pi T}\left(\frac{e^{-v}}{v}\right)\right] / \partial \phi\right\} d v=0
$$

Second term:

$$
f[b(c), c] \frac{d b}{d \phi}=0 \text { because } b=\text { constant }(\infty)
$$

Third term:

$f[a(c), c] \frac{d a}{d \phi}=\frac{1}{4 \pi T}\left[\frac{e^{-u}}{u}\right] \frac{d u}{d \phi}$ where $\frac{d u}{d \phi}=\frac{d\left[\frac{r^{2} \phi}{4 T k}\right]}{d \phi}=\frac{r^{2}}{4 T k}=\frac{u}{\phi}$

therefore:

$$
f[a(c), c] \frac{d a}{d \phi}=\frac{1}{4 \pi T}\left[\frac{e^{-u}}{u}\right] \frac{u}{\phi}=\frac{1}{4 \pi T \phi} e^{-u}
$$

Only term three has a value and: 


$$
\frac{\partial s, t}{\partial \phi}=\sum_{i=1}^{I} \sum_{k=1}^{t} \bar{P}_{i, j, k} q_{t-k+1}
$$

where:

$$
\bar{P}_{i, j, k}=-\frac{1}{4 \pi T \phi} e^{-u_{k}} \quad \text { at } k=1
$$

$$
=-\frac{1}{4 \pi T \phi}\left(e^{-u_{k}}-e^{-u k-1}\right) \quad \text { at } k>1 \ldots \ldots(30)
$$

The partial derivatives of drawdown with respect to transmissivity and effective porosity agree with those shown by McElwee and Yukler, 1978.

Ignoring the higher order terms in eq. 25 the expectation of drawdown can be approximated by eq. 15:

$$
E\left(s_{j, t}\right)=\sum_{i=1}^{I} \sum_{k=1}^{t} \bar{B}_{i, j, k} a_{t-k+1} \ldots \ldots \ldots \ldots
$$

Furthermore, assuming independency of $T$ and $\phi$ the variance of drawdown can be approximated as eq. 16:

$$
\begin{aligned}
\operatorname{var}\left(s_{j, t}\right) & =\left[\frac{\partial s, j, t}{\partial T}\right]^{2} s d t^{2}+\left[\frac{\partial s, j, t}{\partial \phi}\right]^{2} s d s^{2} \\
& =\left[\sum_{i=1}^{1} \sum_{k=1}^{t} \bar{A}_{i, j, k} q_{t-k+1}\right]^{2} s d t^{2}
\end{aligned}
$$




$$
+\left[\sum_{i=1}^{I} \sum_{k=1}^{t} \bar{P}_{i, j, k} q_{t-k+1}\right]^{2} \mathrm{sds}^{2} \ldots \ldots \ldots
$$

where sdt and sds are the standard deviations of the transmissivity and effective porosity respectfully. 
APPENDIX II - Derivation of equation 20

Substituting eq. 16 into eq. 19 , we can express $\sqrt{\operatorname{var}\left(s_{j, t}\right)}$ in terms of unknown pumping Q's more explicitly as:

$$
f(q)=\sqrt{\operatorname{var}(s j, t)}=\sqrt{f t(q)^{2}+f s(q)^{2}} \ldots \ldots \ldots
$$

where:

$$
f t(q)=\sum_{i=1}^{I} \sum_{k=1}^{t}\left[\bar{A}_{i, j, k^{q} t-k+1}\right] s d t
$$

and:

$$
f s(q)=\sum_{i=1}^{I} \sum_{k=1}^{t}\left[\bar{p}_{i, j, k^{q}-k+1}\right] s d s
$$

Eq. 19 is a first order Taylor expansion of eq. 31. The first term on the right-hand side of eq. 19, $f\left(Q_{0}\right)$, is the value of the function $f(q)$ calculated (with eq. 31) by using arbitrarily assumed pumping values, Qo's, in eq. 31. The partial derivative in the second terms of eq. 19 can be found by taking the derivative of eq. 31 with respect to $q$ and is expressed as:

$\left.\frac{\partial f(q)}{\partial q_{t-k+1}}\right|_{Q_{0}}=\frac{1}{f\left(Q_{0}\right)}\left[f t(Q 0) \bar{A}_{i, j, k}(s d t)+f s(Q 0) \bar{P}_{i, j, k}(s d s)\right] \ldots$ 
Substituting eq. 32 into eq. 19 and multiplying it with $q_{t-k+1}$ and Qo $t-k+1$, respectively pe obtain:

$$
\begin{aligned}
f(q)= & f\left(Q_{0}\right) \\
& -\frac{1}{f\left(Q_{0}\right)} \sum_{i=1} \sum_{k=1}^{t}\left[f t\left(Q_{0}\right) \bar{A}_{i, j, k}(s d t)+f s(Q 0) \bar{P}_{i, j, k}(\operatorname{sds})\right] Q_{t-k+1} \\
& +\frac{1}{f\left(Q_{0}\right)} \sum_{i=1} \sum_{k=1}^{t}\left[f t\left(Q_{0}\right) \bar{A}_{i, j, k}(s d t)+f s\left(Q_{0}\right) \bar{P}_{i, j, k}(s d s)\right] q_{t-k+1}
\end{aligned}
$$

( term -3)

$+\mathrm{HOT}$

The second term of eq. 33 cancels the first term as shown.

First, the second term reduces to $f\left(Q_{0}\right)$ as shown:

$$
\begin{aligned}
& \frac{1}{f\left(Q_{0}\right)} \sum_{i=1}^{I} \sum_{k=1}^{t}\left[f t(Q 0) \bar{A}_{i, j, k}(s d t)+f s(Q 0) \bar{P}_{i, j, k}(s d s)\right] Q_{t-k+1} \\
& \text { reduces to } \\
& \frac{f t\left(Q_{0}\right)}{Q_{0}} \quad \underbrace{}_{\frac{f s\left(Q_{0}\right)}{Q_{0}}} \\
& \underbrace{f t\left(Q_{0}\right)^{2}+f s\left(Q_{0}\right)^{2}}
\end{aligned}
$$

Therefore, term $1+$ term $2=f\left(Q_{0}\right)-f\left(Q_{0}\right)=0$

By dropping the higher order terms (HOT) the third term of eq. 33 can be written as eq. 20 . 
Table 1 Effect of Aquifer Parameter Uncertainty on 95\% Reliable Optimal Unsteady Pumping Strategy for Hypothetical Problew (run 1d)

\begin{tabular}{|c|c|c|c|c|c|c|}
\hline \multirow[t]{2}{*}{ Run } & $1 d$ & $1 \varepsilon$ & 28 & 38 & 48 & 58 \\
\hline & \multicolumn{6}{|c|}{ Pumping (L/s) } \\
\hline Day 1 & 96.1 & 85.8 & 70.2 & 51.4 & 85.3 & 83.3 \\
\hline 2 & 90.1 & 76.4 & 63.4 & 47.1 & 74.8 & 70.9 \\
\hline 3 & 84.9 & 70.4 & 59.3 & 44.7 & 68.3 & 63.7 \\
\hline 4 & 80.2 & 66.3 & 56.4 & 43.0 & 64.0 & 59.2 \\
\hline $\mathbf{5}$ & 76.9 & 63.2 & 54.2 & 41.7 & 60.9 & 56.2 \\
\hline 6 & 36.9 & 57.3 & 52.5 & 40.7 & 58.7 & 54.2 \\
\hline 7 & 0.0 & 0.0 & 28.7 & 40.0 & 0.0 & 52.8 \\
\hline 8 & 0.0 & 0.0 & 0.0 & 25.6 & 23.3 & 0.0 \\
\hline Avg. Pumping & 58.1 & 52.4 & 48.1 & 41.8 & 54.4 & 55.0 \\
\hline $\begin{array}{l}\left.\text { Avg. gradient( }()_{1}\right) \\
\text { gradient SD } \\
\text { Sum of sqd. }\end{array}$ & $\begin{array}{l}0.08 \\
0.058\end{array}$ & $\begin{array}{l}0.079 \\
0.043\end{array}$ & $\begin{array}{l}0.085 \\
0.057\end{array}$ & $\begin{array}{l}0.095 \\
0.062\end{array}$ & $\begin{array}{l}0.098 \\
0.061\end{array}$ & $\begin{array}{l}0.14 \\
0.084\end{array}$ \\
\hline head diff. $\left(m^{2}\right)$ & 1.24 & 1.08 & 1.30 & 1.72 & 1.79 & 4.99 \\
\hline $\begin{array}{l}\text { Obj. } \\
\text { func. }\end{array}$ & 15.63 & 13.54 & 15.66 & 19.82 & 21.18 & 55.53 \\
\hline $\begin{array}{c}0 \& 4 \cos t 8 \\
\left(\$ \times 10^{3}\right)\end{array}$ & 2.31 & 1.93 & 1.65 & 1.32 & 1.93 & 1.84 \\
\hline
\end{tabular}

Model Run:

1d. Deterministic model

Transmissivity CV Effective porosity CV
18 .
0.2
0.2
$2 s$.
0.4
0.2
38.
0.8
0.2
48.
0.2
0.4
58.
0.2
0.8 
Table 2 Effect of Aquifer Parameter Uncertainty on $80 \%$ Reliable Optimal Unsteady Pumping Strategy for Hypothetical Problem (run 1d)

\begin{tabular}{|c|c|c|c|c|c|c|}
\hline Run & $1 d$ & 18 & 28 & 38 & 48 & 58 \\
\hline & & & Pumping & $(L / 8)$ & & \\
\hline Day 1 & 96.1 & 94.6 & 85.7 & 69.8 & 93.2 & 90.6 \\
\hline 2 & 90.1 & 86.0 & 76.7 & 63.2 & 85.1 & 82.0 \\
\hline 3 & 84.9 & 78.8 & 71.1 & 59.2 & 77.6 & 74.7 \\
\hline 4 & 80.2 & 73.9 & 67.1 & 56.4 & 72.4 & 69.3 \\
\hline 5 & 76.9 & 70.2 & 64.1 & 54.3 & 68.7 & 65.6 \\
\hline 6 & 36.9 & 21.5 & 44.9 & 52.7 & 36.2 & 63.0 \\
\hline 7 & 0.0 & 0.0 & 0.0 & 20.1 & 0.0 & 0.0 \\
\hline 8 & 0.0 & 0.0 & 0.0 & 0.0 & 0.0 & 4.9 \\
\hline Avg. Pumping & 58.1 & 53.1 & 51.2 & 47.0 & 54.1 & 56.3 \\
\hline $\begin{array}{l}\text { Avg. gradient( }(x) \\
\text { gradient } S D \\
\text { Sum of sqd. }\end{array}$ & $\begin{array}{l}0.08 \\
0.058\end{array}$ & $\begin{array}{l}0.067 \\
0.047\end{array}$ & $\begin{array}{l}0.070 \\
0.048\end{array}$ & $\begin{array}{l}0.076 \\
0.050\end{array}$ & $\begin{array}{l}0.076 \\
0.049\end{array}$ & $\begin{array}{l}0.097 \\
0.060\end{array}$ \\
\hline head diff. $\left(\mathrm{m}^{2}\right)$ & 1.24 & .77 & .85 & 1.04 & 1.01 & 1.70 \\
\hline $\begin{array}{l}\text { Obj. } \\
\text { func. }\end{array}$ & 15.63 & 10.37 & 11.03 & 12.80 & 12.89 & 20.36 \\
\hline $\begin{array}{c}0 \& \cos t 8 \\
\left(\$ \times 10^{3}\right)\end{array}$ & 2.31 & 2.04 & 1.89 & 1.62 & 2.04 & 2.06 \\
\hline
\end{tabular}


Table 3 Summary of Trends Produced by Stochastic Analys is (hydraulic objective function)

Value affected Increased reliability in trans. in eff. por.

1. Influence coef.

used with:

$\begin{array}{lll}\text { objec. func. } & \text { decrease } & \text { large decr. small decr. } \\ \text { DD constraint } & \text { increase } & \text { large incr. small incr. }\end{array}$

2.Daily pumping

decreases

large decr. small decr.

3.Total pumping

decreases

large decr. small incr.

4. Gradient (reverse)

steeper \&

steeper \& less smooth

less smooth

5.0bj. func. value increase

small incr. large incr. 


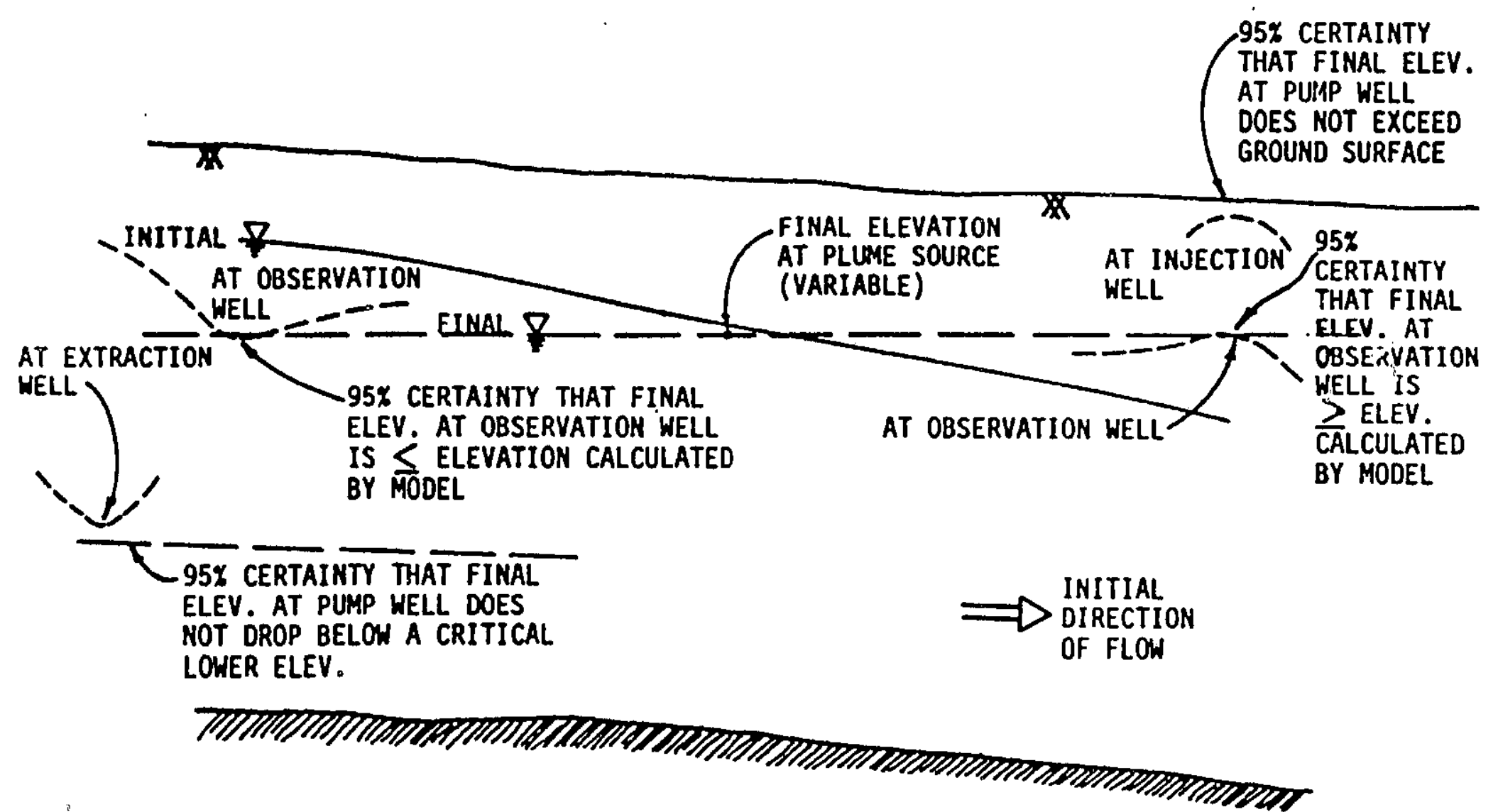

Figure 1. Cross-section Demonstrating Sample Stochastic Constraints on Final Water Levels 


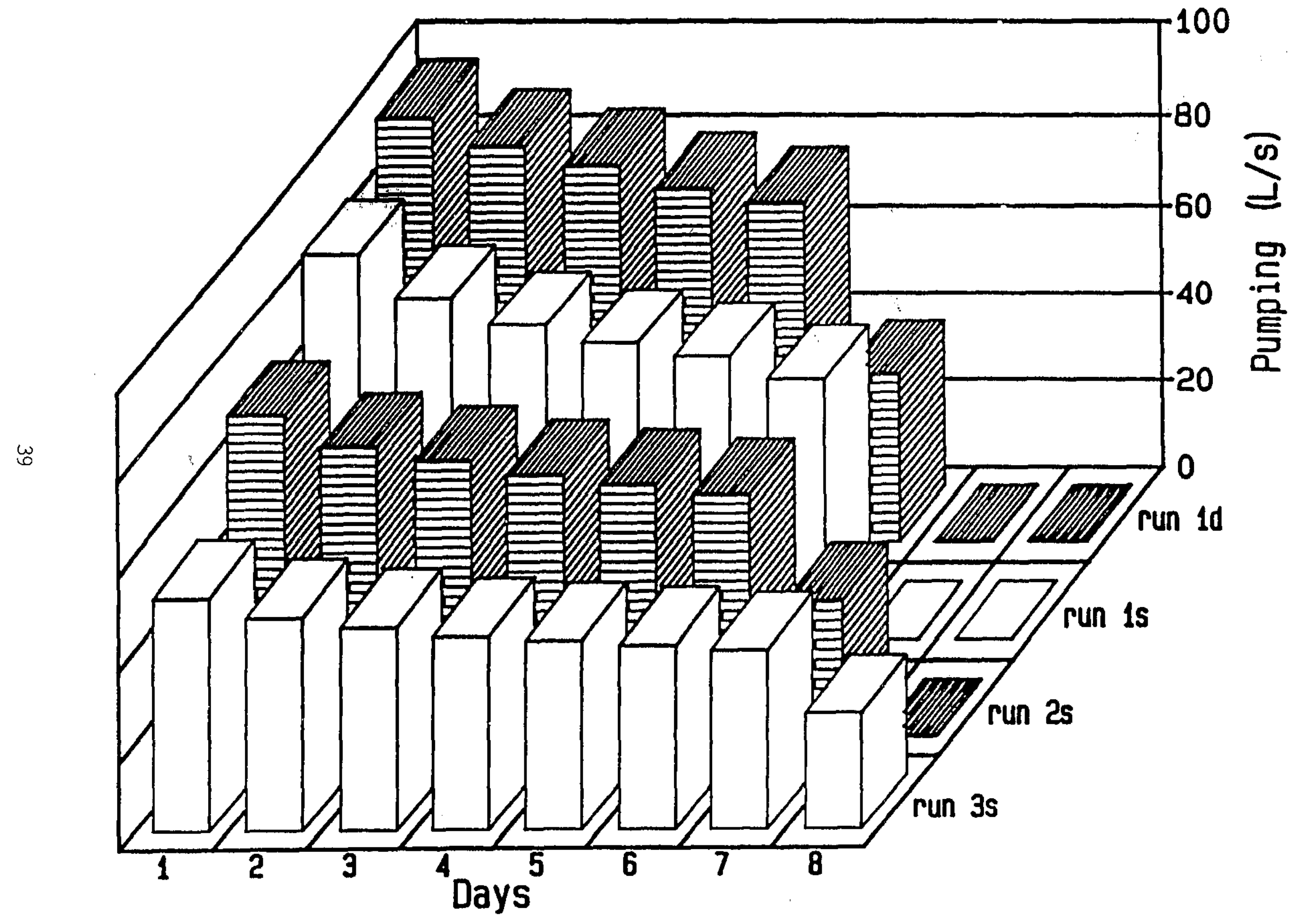

Figure 2. Daily Pumping strategies for Increasing Uncertainty of Transmissivity as Compared to the Deterministic Strategy (95\% Reliability Level) 


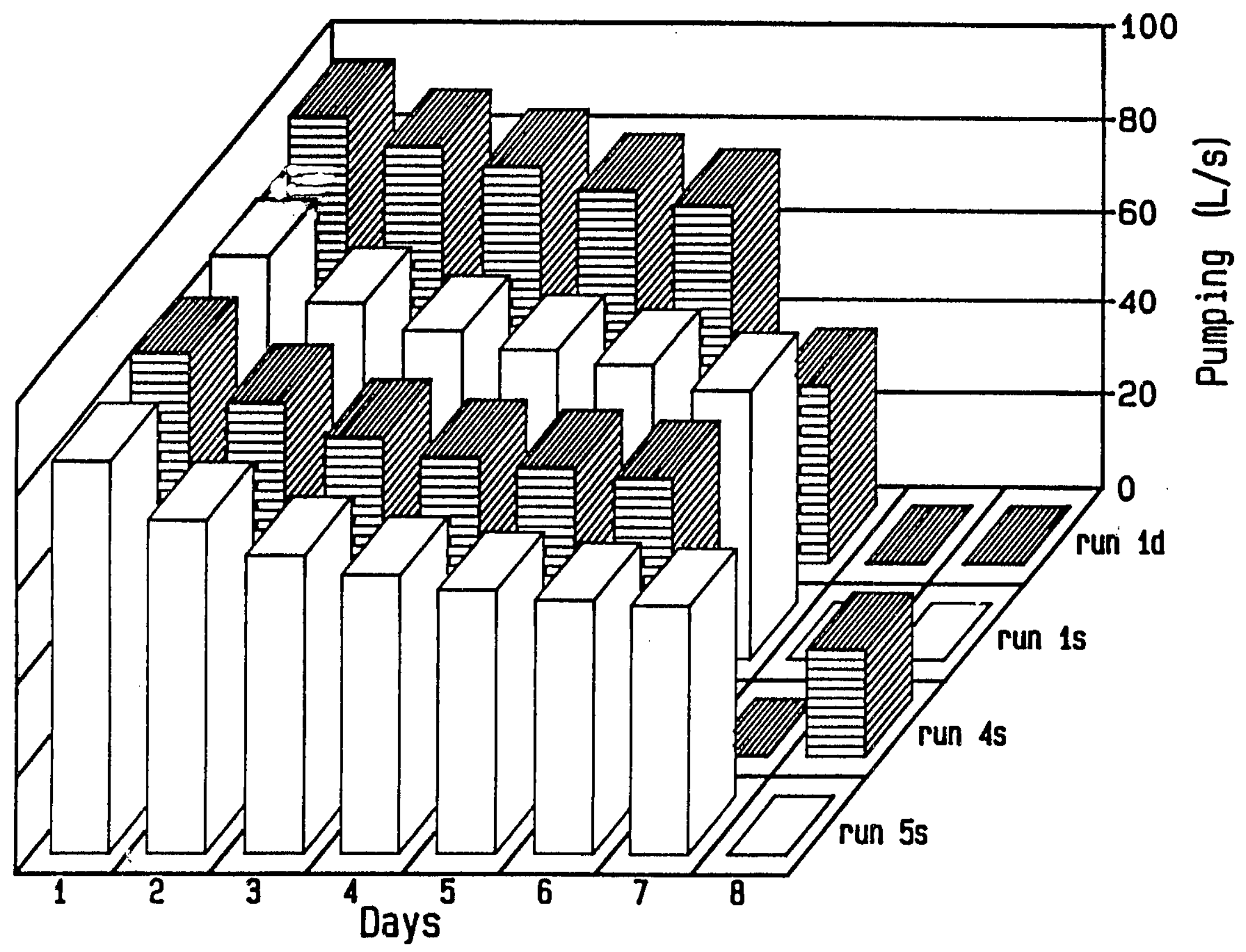

Figure 3. Daily Pumping strategies for Increasing Uncertainty of Effective Porosity as Compared to the Deterministic Strategy (95\% Reliability Level) 


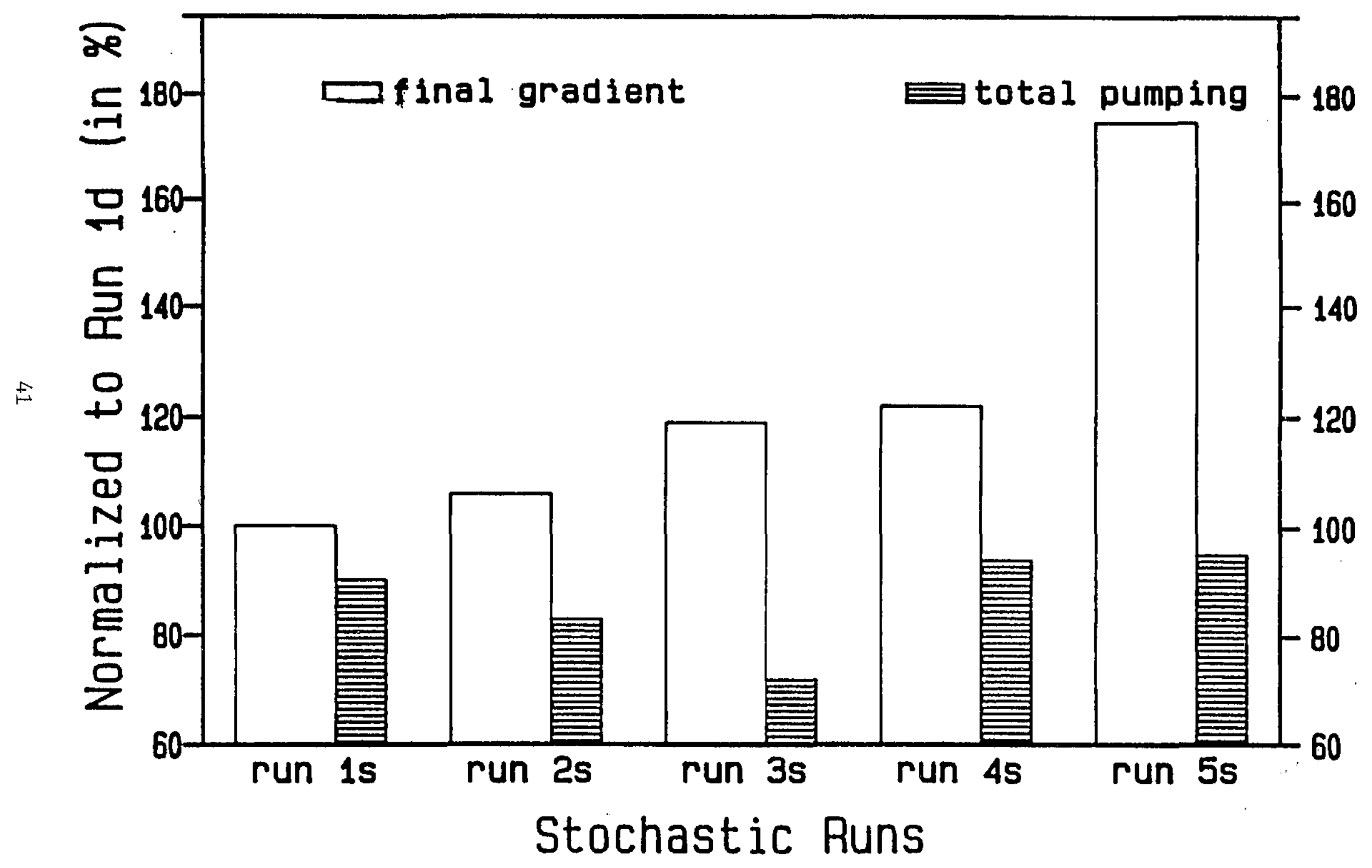

Figure 4. Comparisons of Final Gradient and Total Pumping for the Stochastic Runs at the 95\% Reliability Level 


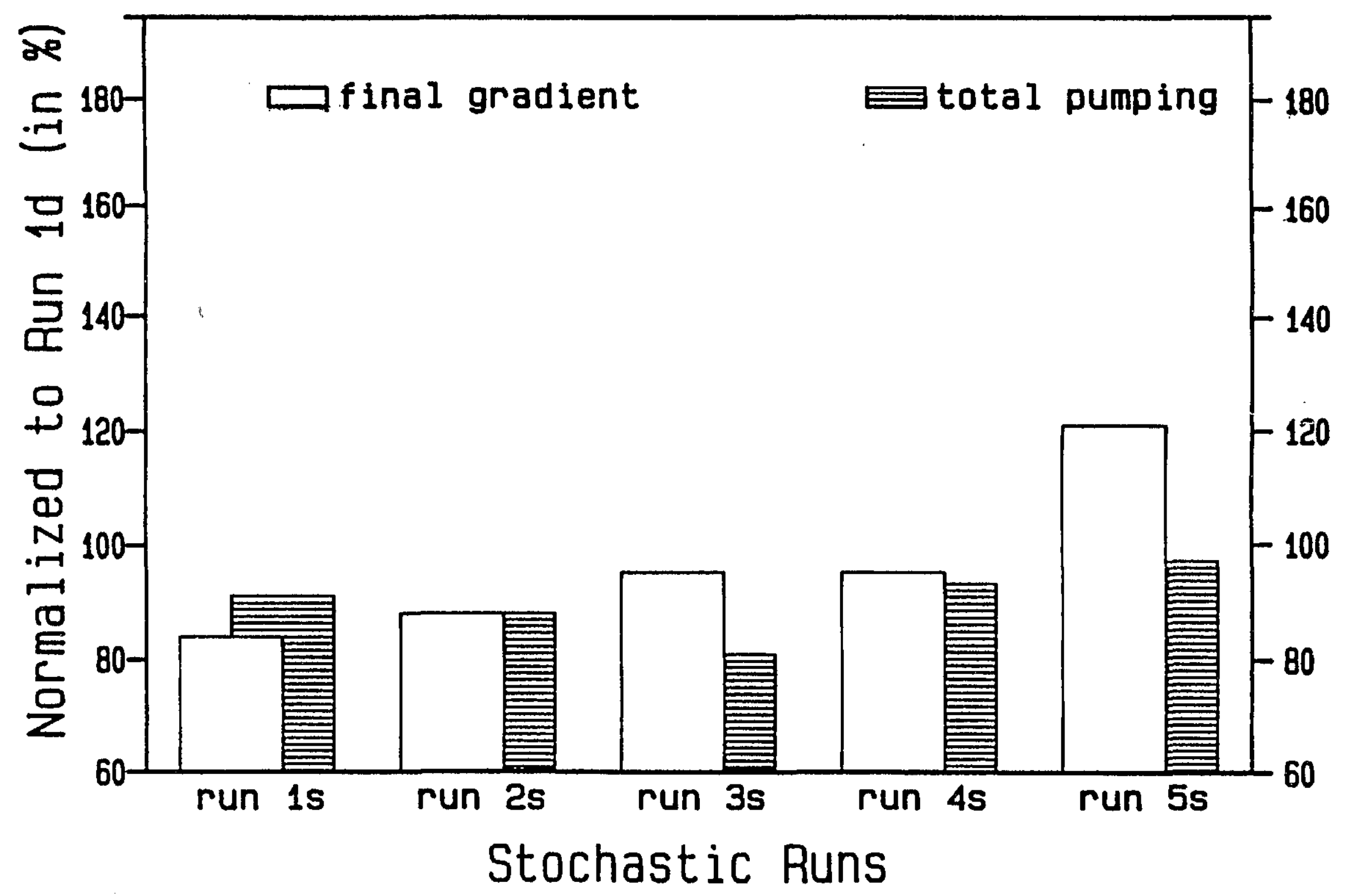

Figure 5. Comparisons of Final Gradient and Total Pumping for the Stochastic Runs at the $80 \%$ Reliability Level 